\title{
Continuous Veno-Venous Hemofiltration (CVVH) Improves Iron Metabolism Disorders in Patients With Sepsis : A Single-Center Prospective Cohort Study
}

\section{Meng-Meng An}

First Affiliated Hospital of Dalian Medical University

Yi Jiang

General Hospital of Tianjin Medical University

Bei-Bei Jin

First Affiliated Hospital of Dalian Medical University

Da Cao

First Affiliated Hospital of Dalian Medical University

Ping Gong ( $\square$ gongp828@sina.cn )

First Affiliated Hospital of Dalian Medical University https://orcid.org/0000-0001-6813-8186

\section{Original research}

Keywords: sepsis, continuous veno-venous hemofiltration, iron metabolism, hepcidin, IL-6

Posted Date: December 9th, 2020

DOI: https://doi.org/10.21203/rs.3.rs-122150/v1

License: (c) (i) This work is licensed under a Creative Commons Attribution 4.0 International License.

Read Full License 


\section{Abstract}

Background: Iron metabolism disorder is commonly seen in patients with sepsis. This study aimed to evaluate whether continuous veno-venous hemofiltration (CVVH) improved the iron metabolism disorders in sepsis.

Methods: In a single-center, retrospective cohort study, totally 89 sepsis patients were prospectively enrolled and divided into the CVVH group ( $n=39)$ and the control group $(n=50)$. Clinical and laboratory data were collected and compared between the groups on days 1, 3 and 7 of ICU admission. Plasma interleukin (IL)-6, hepcidin, erythropoietin (EPO), ferritin and soluble transferrin receptor (sTfR) were determined by enzyme linked immunosorbent assay (ELISA). Sequential organ failure scores (SOFA) on days 1 and 7, and 28-day survival between groups were compared.

Results: Plasma IL-6, hepcidin, ferritin and RDW on days 3 and 7 were significantly reduced in the CVVH group compared with those in the control group (all $P<0.05$ ). The CVVH group had a significantly lower SOFA score on day 7 compared with the control group $(P<0.05)$. Hemoglobin and EPO were gradually decreased within the first week of ICU admission in both groups although no significant differences between the groups were observed. There was no significant difference in STfR between the two groups along with the time (all $P>0.05$ ). In addition, there were no significant differences in 28-day survival rate and median survival time between the two groups.

Conclusion: $\mathrm{CVVH}$ improves iron metabolism disorders and the disease severity in sepsis. However, it does not alleviate anemia and fails to improve the survival.

\section{Background}

Sepsis with an in-hospital mortality rate of $25.8 \%$ has so far been one of the leading causes of death in critically ill patients [1]. Anemia is commonly observed in patients with sepsis, which is mostly due to the involvement of inflammatory mediators $[2,3]$. The release of inflammatory mediators were associated with elevated plasma hepcidin, leading to iron metabolism disorders and subsequent anemia in severe sepsis [3]. Interleukin (IL)-6 is a key inflammatory cytokine which induces an abruptly increased synthesis of hepcidin and decreases plasma iron in patients with sepsis [4]. The survival of sepsis was closely related with the indicators reflecting iron metabolism disorders and inflammation-associated anemia. We previously observed that plasma iron, hemoglobin and soluble transferrin receptor (sTfR)/log ferritin were significantly decreased and the plasma erythropoietin (EPO), sTfR, hepcidin, ferritin and IL-6 were significantly increased in patients with sepsis within the first week on intensive care unit (ICU) admission. More importantly, the plasma EPO, hepcidin, ferritin and IL-6 were associated with the 28-day survival [4].

No effective therapies have been developed so far for the treatment of severe inflammation-associated anemia and related iron metabolism disorders in sepsis. Conventional blood transfusion and iron supplement are not routinely recommended in the current clinical practice. Blood transfusion was reported to be associated with higher mortality and also failed in anemia correction in critically ill patients 
$[5,6]$. Although enteral iron supplementation (ferrous sulfate $325 \mathrm{mg}$ three times daily) reduced transfusion rate in critically ill patients with baseline iron deficiency, iron is a toxic compound which may induce oxidative stress and bacterial growth $[7,8]$. In addition, the optimal route of iron administration still need to be determined [7].

Continuous venous-venous hemofiltration ( $\mathrm{CVVH}$ ) as a commonly used mode of continuous blood purification is a pivotal treatment strategy for renal failure [9-14]. CVVH can also non-selectively remove endotoxin and inflammatory mediators, rebalance acid-base disorders, regulate immune stability, and maintain stable environment through the mechanisms of diffusion, convection and adsorption [15]. It is therefore applicable for eliminating inflammatory mediators in sepsis [16]. However, the impact of CVVH on iron metabolism disorders in patients with sepsis remains unclear. This prospective study aimed to determine the effect of $\mathrm{CVVH}$ on iron metabolism disorders in patients with sepsis.

\section{Methods}

\section{Ethics statement}

This study was performed in accordance with the Declaration of Helsinki adopted by the World Medical Association (2013 edition) [17]. The study protocol was approved by the Medical Ethics Committee of the First Affiliated Hospital of Dalian Medical University (PJ-KS-KY-2020-138), Dalian, China. A written informed consent was obtained from all participants or legal guardians at the time of initial admission.

\section{Patients}

Patients diagnosed with sepsis and admitted to the ICU at the First Affiliated Hospital of Dalian Medical University from October 1, 2015 to December 30, 2017, were prospectively collected. All included patients met the diagnostic criteria of the third international consensus definitions for sepsis and septic shock (Sepsis-3), accompanied by sepsis-induced acute kidney injury with increase in creatinine or oliguria with fluid overload, and hyperkalemia [10-12, 18-20]. The exclusion criteria were as follows: less than 18 years old; pregnancy; with stomach cancer and acute bleeding; with blood system diseases [e.g., leukemia, myeloma and red blood cell diseases, including polycythemia vera, abnormal mean red blood cell volume ( $\mathrm{MCV}<80.8$ or $\left.>99.2 \mu \mathrm{m}^{3}\right)$, abnormal mean hemoglobin content $(\mathrm{MCH}<26.4$ or $>34.2 \mathrm{pg}$ ) or abnormal mean hemoglobin concentration ( $\mathrm{MCHC}<32.0$ or $>35.4 \mathrm{~g} / \mathrm{dL}$ )]; with acute or chronic renal insufficiency or renal failure requiring hemodialysis; with liver cirrhosis; prior blood transfusion within 1 week before admission or during hospitalization; with anemia treated with iron and erythropoietin within 3 months before admission.

The enrolled patients with sepsis were divided into two groups. Patients in the CVVH group received $\mathrm{CVVH}$ treatment while those in the control group did not receive CVVH treatment due to refusal either from the patient or from legal guardians.

\section{Treatment}


The control group received routine treatment according to the Surviving Sepsis Campaign guidelines, including intensive monitoring, fluid resuscitation, oxygen administration or mechanical ventilation, antimicrobial therapy (blood culture was conducted before the administration of antibiotics), vasopressor administration if necessary, glucose control, diuretics for oliguria with fluid overload, etc [21].

Patients in the CVVH group underwent CVVH (Golden Prisma Flex, Sweden) within $24 \mathrm{~h}$ after diagnosis in addition to the routine treatment. A 11.5Fr double lumen hemofiltration catheter (Lily Technology, Guangdong, China) was percutaneously inserted into the femoral vein or the internal jugular vein. Hemofiltration was performed using the PrimaFlex apparatus (ALPRI, France) equipped with an acrylonitrile and sodium methallyl sulfonate copolymer hollow-fiber high-flux hemofilter $\left(1.5 \mathrm{~m}^{2}\right.$, Braun Diapact, Germany), and was delivered in CVVH mode. CVVH was performed at a dose of $35 \mathrm{~mL} / \mathrm{kg} / \mathrm{h}$ for $72 \mathrm{~h}$ [22], and thereafter for $12 \mathrm{~h}$ per day, depending on the condition of the patient. The average blood flow rates were $150-200 \mathrm{~mL} / \mathrm{min}$ and the dehydration volume was $300-380 \mathrm{~mL} / \mathrm{h}$. The hemofilter was changed every $48 \mathrm{~h}$. The bicarbonate replacement solutions were commercially prepared by Qingshan Likang, Pharmaceutical Co. (Chengdu, China), and was pre-hemofilter diluted. Unfractionated heparin as an anticoagulant or no heparinization was used. The dosage of the unfractionated heparin was adjusted according to the patient's blood coagulation state.

\section{Data collection}

The following clinical data were collected upon admission: gender, age, sites of infection, comorbidities, numbers of post-operative patients, patients using vasopressors and patients receiving mechanical ventilation, laboratory results, and survival time within 28 days. Hemoglobin, red blood cell distribution width (RDW), reticulocytes, mean corpuscular volume, mean corpuscular hemoglobin, and mean corpuscular hemoglobin concentration were measured by an automatic blood cell analyzer (XN-2000; Sysmex, Kobe, Japan). Sequential organ failure assessment (SOFA) scores were assessed on ICU admission based on age, medical history, vital signs, and laboratory results.

Peripheral venous blood $(5 \mathrm{~mL})$ was drawn on days 1 (before CVVH treatment), 3 and 7 of admission, centrifuged (TGL-20MS, $4^{\circ} \mathrm{C}, 2000 \times g$ ) for $10 \mathrm{~min}$, and stored at $-80^{\circ} \mathrm{C}$ for later analysis. Plasma IL-6, hepcidin (Uscn Life Sciences, Wuhan, China), EPO, ferritin (Abcam, Cambridge, MA, USA) and sTfR (R\&D Systems, MN, USA) were detected by enzyme linked immunosorbent assay (ELISA).

\section{Statistical analyses}

Statistical analyses were performed using SPSS 23.0 (IBM, Armonk, NY, USA). Continuous variables are expressed as mean \pm standard deviation (SD) for normal distribution, or median and range for skewed distribution. Categorical variables were compared using Pearson's chi-squared or Fisher's exact test. Continuous variables were compared by Mann-Whitney $U$ test or $t$ test. Cumulative survival curves were depicted using the Kaplan-Meier method and compared by log-rank test. $P<0.05$ was considered to be statistically significant. 


\section{Results}

\section{Baseline characteristics}

Totally 127 patients with sepsis were initially enrolled and 89 patients were finally included in the study (Figure 1). The CVVH group included 39 patients while 50 patients were included in the control group. No significant differences of baseline characteristics including age, male, sites of infection, comorbidities, the numbers of postoperative patients, patients using vasopressors and patients receiving mechanical ventilation, $\mathrm{PaO}_{2} / \mathrm{FiO}_{2}$, procalcitonin, lactate, creatinine, reticulocytes, mean corpuscular volume, mean corpuscular hemoglobin, mean corpuscular hemoglobin concentration, and SOFA scores were detected between the groups (Table 1).

\section{The effect of CVVH on plasma IL-6}

On admission, plasma IL-6 level did not differ between the two groups ( $P>0.05$, Figure 2$)$. Significantly decreased IL-6 level from day 1 to days 3 and 7 was observed in both groups (all $P<0.05$ ). However, plasma IL- 6 on days 3 and 7 in the CVVH group were significantly lower than that in the control group (both $P<0.05$ ).

\section{The effect of CVVH on hemoglobin, plasma EPO and RDW}

There were no significant differences in hemoglobin, plasma EPO and RDW between groups upon admission (all $P>0.05$ ). However, both groups presented with gradually decreased hemoglobin and EPO within the first week of ICU admission without significant differences between the groups (all $P<0.05$, Figures $3 \mathrm{~A}$ and $3 \mathrm{C}$ ). RDW on days 3 and 7 in both groups were higher than that on day 1 without significant difference between days 3 and 7 (both $P<0.05$, Figure $3 \mathrm{~B}$ ). However, the CVVH group had a significantly lower RDW on days 3 and 7 than the control group (both $P<0.05$ ).

\section{The effect of CVVH on iron metabolism disorder}

Plasma hepcidin and ferritin did not differ between the groups upon admission (all $P>0.05$, Figure 3 ). However, plasma hepcidin on days 3 and 7 in both groups were lower than that on day 1 (all $P<0.05$, Figure 3D). Plasma hepcidin on days 3 and 7 in the CVVH group was significantly reduced compared with that in the control group (both $P<0.05$ ). Plasma ferritin on days 3 and 7 in both groups were higher than that on day 1 (all $P<0.05$, Figure 3E) while plasma ferritin on days 3 and 7 in the CVVH group was significantly lower than that in the control group (both $P<0.05$ ). There were no significant differences in plasma sTfR between the two groups, or among different time points (all $P>0.05$, Figure 3F).

\section{The effect of CVH on the severity and outcome}

SOFA scores significantly decreased from day 1 to day 7 in both groups (all $P<0.05$ ). However, SOFA score on day 7 in the CVVH group was significantly lower than that in the control group $(P<0.05)$. 
Survival rate did not differ between the CVVH and the control groups $(71.8 \%$ vs $62.0 \%, P=0.332)$, nor did the median survival time $[28$ days $(95 \% \mathrm{Cl} 20.8-25.7)$ versus 28 days $(95 \% \mathrm{Cl} 17.1-22.8), P=0.187$, Figure 4].

\section{Discussion}

The present study evaluated the impact of $\mathrm{CVVH}$ on dynamic changes of indicators reflecting the iron metabolism disorder in sepsis patients. We found that plasma IL-6, hepcidin, ferritin and RDW was significantly increased in sepsis but reduced after CVVH treatment. However, there was no significant difference in STfR between the two groups along with the time. In addition, $\mathrm{CVVH}$ did not seem to improve the 28-day survival for patients with sepsis.

Plasma hepcidin, IL-6, ferritin and STfR are inflammatory anemia-associated parameters for patients with sepsis. Our previous study on sepsis observed that these parameters increased remarkably within the first week of ICU admission [4]. Furthermore, plasma IL-6 was positively correlated with plasma hepcidin and ferritin in patients with sepsis [4]. Hepcidin as a liver-producing peptide hormone plays a central role in regulating plasma iron [23]. It inhibits iron absorption from duodenum, release from hepatocytes and circulating macrophages, resulting in a low level of plasma iron [24]. The production of hepcidin is affected by inflammation. IL-6 can induce up-regulation of hepcidin expression through the STAT-3 signaling pathway, thus causes a series of disorders in iron metabolism, which were significantly associated with anemia and 28-day mortality [2-4, 7, 25-28]. The current study revealed that CVVH treatment significantly reduced plasma IL-6, hepcidin and ferritin, supporting previous findings that inflammatory mediators such as plasma IL- 6 and TNF- $a$ can be cleared by hemofiltration in animal and human experiments $[10,13,16,29]$. The mechanism that CVVH clears plasma IL- 6 may be adsorption rather than convection because IL- 6 with a molecular weight of $26 \mathrm{kDa}$ is difficult to be filtered through the pores of the membrane in $\mathrm{CVVH}$ [29]. In addition, polymyxin B in the filtration membrane is a cyclic lipophilic peptide antibiotic which has high affinity for the lipid A moiety in the endotoxin, and thus CVVH can effectively adsorb lipopolysaccharide. Rapid removal of lipopolysaccharide as an activator of cytokines production by CVVH can also lead to a decreased production of IL-6 [29]. Moreover, hepcidin with a molecular weight of approximately $10 \mathrm{kDa}$ can be effectively removed through the membrane in $\mathrm{CVVH}$ [30]. The reduction of plasma hepcidin and ferritin in sepsis patients treated by CVVH may also be associated with the reduction of plasma IL- 6 because the clearance of IL- 6 by CVVH can relieve the upregulation of hepcidin through IL-6-hepcidin axis and block the induction of pro-inflammatory cytokines such as TNF- $\alpha$, IL-1 $\beta$ and IL- 6 to the production of ferritin [25-27].

sTfR level seemed to be unaffected by CVVH in patients with sepsis in this study. sTfR has a large molecular weight of $85 \mathrm{kDa}$ with a very low CVVH clearance rate. Moreover, sTfR reflects the degree of iron availability for cells, but is not affected by inflammation. Therefore, the clearance of IL- 6 by CVVH does not affect the production of sTfR. 
Plasma EPO was reported to be elevated with a tendency of gradual decrease within the first week of ICU admission in sepsis patients [4]. However, we found in the present study that there was no significant change in EPO concentration in sepsis patients treated by CVVH. This may be mainly because EPO with a molecular weight of $34 \mathrm{kDa}$ is difficult to be filtered through the membrane pores in $\mathrm{CVVH}$ [31]. In addition, CVVH and EPO production is mutually affected. Plasma EPO is usually increased in response to anemia and hypoxemia, whereas the production of EPO is compromised by impaired renal function (which decreases EPO production) and pro-inflammatory cytokines (such as TNF-a and IL-6, which inhibits EPO production) in patients with sepsis [4, 32]. On the one hand, CVVH may ameliorate hypoxemia and acute lung injury, thus decreasing the production of EPO [33]. However, clearance of proinflammatory cytokines by CVVH may increase the production of EPO. Considering the complicated effects of CVVH on EPO production, it is not surprising to see in this study that CVVH failed to affect plasma EPO concentration in sepsis patients.

RDW is typically used as part of a complete blood count to quantify changes in the size of circulating red blood cells [34]. For patients with sepsis, significantly increased RDW is observed on admission and can serve as a useful predictor of mortality, as evidenced in our previous study [4, 35]. In the present study, we found that sepsis patient treated with CVVH had a lower RDW. The mechanism that CVVH affects RDW remains unknown. However, previous research displayed a strong correlation between RDW and IL-6, and IL-6 was one of independent predictors of RDW [4, 34]. Pro-inflammation cytokines such as IL-6 can also impair the maturation of RBC and thus accelerate the entrance of immature RBCs into circulation. Therefore, we speculate that the decreased RDW by CVVH may be related to the clearance of IL- 6 .

Hemoglobin level in sepsis patients treated with CVVH did not change greatly within the first week. The incidence of sepsis-related anemia is as high as $90 \%$ within 3 days. It is caused by multiple mechanisms including infection, iatrogenic blood loss, dilution during fluid resuscitation, decreased plasma iron, inhibition of erythropoietin production, shortened red blood cell life, and malnutrition with infection to be the major reason [3, 36-38]. Inflammation up-regulated hepcidin which inhibited plasma iron levels and had a measurable effect on hemoglobin levels from 7 days after diagnosis of sepsis [23]. Therefore, within the first week, the effect of decreased hepcidin by CVVH on hemoglobin production may be insignificant.

The 28-day survival of sepsis failed to be improved by CVVH, which is inconsistent with the metaanalysis published by Putzu et al.[14]. There are several explanations for this result. First, the relatively small sample size of this study may not be adequate to reach a statistically significant result. A multicenter randomized trial with a large sample size would be necessary in the future trials. Second, iron metabolism disorder partly contributes to the severity of sepsis and may be insufficient to affect the outcome. Many other complications accompanied by sepsis may also influence the mortality.

There are several other limitations in the present study. First, this is a single-center study, which inevitably introduces selection bias. Second, CVVH is not a standardized technique for the treatment of sepsis. Therefore, the CVVH regimen and system used could affect the results. Third, we did not assess the 
differences in subgroups of sex and septic shock. However, the sex distribution and the number of patients using vasopressors on admission was balanced between the groups. Finally, the implement of the $\mathrm{CVVH}$ received by patients with sepsis was dependent on the choice of these patients or their legal guardians except the decision of their doctor-in- charge. Hence, this might produce a selection bias.

\section{Conclusions}

CVVH improves iron metabolism disorders and the disease severity in sepsis. However, it does not alleviate anemia and fails to improve the survival.

\section{Abbreviations}

ICU: intensive care unit; Cl: Confidence interval; CVVH: Continuous veno-venous hemofiltration; ELISA: enzyme linked immunosorbent assay; EPO: erythropoietin; IL-6: interleukin-6; MCV: mean red blood cell volume; $\mathrm{MCH}$ : mean hemoglobin content; $\mathrm{MCHC}$ : mean hemoglobin concentration; RBC: Red blood cells; RDW: red blood cell distribution width; SOFA: Sequential organ failure scores; sTfR: soluble transferrin receptor; TNF-a:Tumor Necrosis Factor-a.

\section{Declarations}

\section{Ethics approval and consent to participate}

The study protocol was approved by the Medical Ethics Committee of the First Affiliated Hospital of Dalian Medical University (PJ-KS-KY-2020-138). Written informed consent was obtained from all patients (or their relatives) upon their initial admission to the hospital and from healthy volunteers.

\section{Consent for publication}

Not applicable.

\section{Availability of data and material}

The datasets analyzed during the current study are available from the corresponding author on reasonable request.

\section{Competing interests}

The authors declare that they have no competing interests.

\section{Funding}

This study was supported by the National Natural Science Foundation of China (81571869).

\section{Authors' contributions}


PG conceived and designed the experiments. M-MA, YJ, B-BJ, and DC carried out the experiments. M-MA, YJ and PG analyzed the data. M-MA and PG wrote the manuscript. PG took overall responsibility for the manuscript. All authors approved the final version of the manuscript.

\section{Acknowledgments}

The authors sincerely appreciate Dong-Dong Zhou for his technical assistance and the staff of Emergency Intensive Care Unit for their helpful contributions.

\section{References}

1. Vincent JL, Marshall JC, Namendys-Silva SA, Francois B, Martin-Loeches I, Lipman J, et al. Assessment of the worldwide burden of critical illness: the intensive care over nations (ICON) audit. The Lancet Respiratory medicine. 2014; 2(5):380-6.

2. Loftus TJ, Mira JC, Stortz JA, Ozrazgat-Baslanti T, Ghita GL, Wang Z, et al. Persistent inflammation and anemia among critically ill septic patients. J Trauma Acute Care Surg. 2019; 86(2):260-7.

3. Weiss G, Ganz T, Goodnough LT. Anemia of inflammation. Blood. 2019; 133(1):40-50.

4. Jiang $Y$, Jiang FQ, Kong F, An MM, Jin BB, Cao D, Gong P. Inflammatory anemia-associated parameters are related to 28-day mortality in patients with sepsis admitted to the ICU: a preliminary observational study. Annals of intensive care. 2019; 9(1):67.

5. Vincent JL, Baron JF, Reinhart K, Gattinoni L, Thijs L, Webb A, et al. Anemia and blood transfusion in critically ill patients. JAMA. 2002; 288(12):1499-507.

6. Carson JL, Stanworth SJ, Roubinian N, Fergusson DA, Triulzi D, Doree C, Hebert PC. Transfusion thresholds and other strategies for guiding allogeneic red blood cell transfusion. Cochrane Database Syst Rev. 2016; 10:CD002042.

7. Heming N, Montravers P, Lasocki S. Iron deficiency in critically ill patients: highlighting the role of hepcidin. Crit Care. 2011; 15(2):210.

8. van Iperen CE, Gaillard CA, Kraaijenhagen RJ, Braam BG, Marx JJ, van de Wiel A. Response of erythropoiesis and iron metabolism to recombinant human erythropoietin in intensive care unit patients. Crit Care Med. 2000; 28(8):2773-8.

9. Rhodes A, Evans LE, Alhazzani W, Levy MM, Antonelli M, Ferrer R, et al. Surviving Sepsis Campaign: International Guidelines for Management of Sepsis and Septic Shock: 2016. Crit Care Med. 2017; 45(3):486-552.

10. Romagnoli S, Ricci Z, Ronco C. CRRT for sepsis-induced acute kidney injury. Curr Opin Crit Care. 2018; 24(6):483-92.

11. Hanafusa N. Application of Continuous Renal Replacement Therapy: What Should We Consider Based on Existing Evidence? Blood purification. 2015; 40(4):312-9.

12. Bagshaw SM, Cruz DN, Gibney RT, Ronco C. A proposed algorithm for initiation of renal replacement therapy in adult critically ill patients. Crit Care. 2009; 13(6):317. 
13. Villa G, Zaragoza JJ, Sharma A, Neri M, De Gaudio AR, Ronco C. Cytokine removal with high cut-off membrane: review of literature. Blood purification. 2014; 38(3-4):167-73.

14. Putzu A, Fang MX, Boscolo Berto M, Belletti A, Cabrini L, Cassina T, Landoni G. Blood purification with continuous veno-venous hemofiltration in patients with sepsis or ARDS: a systematic review and meta-analysis. Minerva anestesiologica. 2017; 83(8):867-77.

15. Venkataraman R, Subramanian S, Kellum JA. Clinical review: extracorporeal blood purification in severe sepsis. Crit Care. 2003; 7(2):139-45.

16. Bellomo R, Ronco C. Continuous haemofiltration in the intensive care unit. Crit Care. 2000; 4(6):33945.

17. Hellmann F, Verdi M, Schlemper BR, Jr., Caponi S. 50th anniversary of the Declaration of Helsinki: the double standard was introduced. Archives of medical research. 2014; 45(7):600-1.

18. Singer M, Deutschman CS, Seymour CW, Shankar-Hari M, Annane D, Bauer M, et al. The Third International Consensus Definitions for Sepsis and Septic Shock (Sepsis-3). JAMA. 2016; 315(8):801-10.

19. Dellinger RP, Levy MM, Rhodes A, Annane D, Gerlach H, Opal SM, et al. Surviving sepsis campaign: international guidelines for management of severe sepsis and septic shock: 2012. Crit Care Med. 2013; 41(2):580-637.

20. Thomas ME, Blaine C, Dawnay A, Devonald MA, Ftouh S, Laing C, et al. The definition of acute kidney injury and its use in practice. Kidney international. 2015; 87(1):62-73.

21. Rhodes A, Evans LE, Alhazzani W, Levy MM, Antonelli M, Ferrer R, et al. Surviving Sepsis Campaign: International Guidelines for Management of Sepsis and Septic Shock: 2016. Intensive Care Med. 2017; 43(3):304-77.

22. Joannes-Boyau O, Honore PM, Perez P, Bagshaw SM, Grand H, Canivet JL, et al. High-volume versus standard-volume haemofiltration for septic shock patients with acute kidney injury (IVOIRE study): a multicentre randomized controlled trial. Intensive Care Med. 2013; 39(9):1535-46.

23. van Eijk LT, Kroot JJ, Tromp M, van der Hoeven JG, Swinkels DW, Pickkers P. Inflammation-induced hepcidin-25 is associated with the development of anemia in septic patients: an observational study. Crit Care. 2011; 15(1):R9.

24. Ganz T, Nemeth E. Hepcidin and iron homeostasis. Biochim Biophys Acta. 2012; 1823(9):1434-43.

25. Drakesmith H, Prentice AM. Hepcidin and the iron-infection axis. Science. 2012; 338(6108):768-72.

26. Darveau M, Denault AY, Blais N, Notebaert E. Bench-to-bedside review: iron metabolism in critically ill patients. Crit Care. 2004; 8(5):356-62.

27. Nemeth E, Rivera S, Gabayan V, Keller C, Taudorf S, Pedersen BK, Ganz T. IL-6 mediates hypoferremia of inflammation by inducing the synthesis of the iron regulatory hormone hepcidin. The Journal of clinical investigation. 2004; 113(9):1271-6.

28. Pietrangelo A, Dierssen U, Valli L, Garuti C, Rump A, Corradini E, et al.STAT3 is required for IL-6-gp130dependent activation of hepcidin in vivo. Gastroenterology. 2007; 132(1):294-300. 
29. Peng Y, Yuan Z, Li H. Removal of inflammatory cytokines and endotoxin by veno-venous continuous renal replacement therapy for burned patients with sepsis. Burns. 2005; 31(5):623-8.

30. Zaritsky J, Young B, Gales B, Wang HJ, Rastogi A, Westerman M, et al. Reduction of serum hepcidin by hemodialysis in pediatric and adult patients. Clin J Am Soc Nephrol. 2010; 5(6):1010-4.

31. French C. Erythropoietin in Critical Illness and Trauma. Critical care clinics. 2019; 35(2):277-87.

32. Morceau F, Dicato M, Diederich M. Pro-inflammatory cytokine-mediated anemia: regarding molecular mechanisms of erythropoiesis. Mediators Inflamm. 2009; 2009:405016.

33. Chen J, Chen G, Xiao D, Peng W, Yu G, Lin Y, Zheng F. Continuous venovenous hemofiltration decreases mortality and ameliorates acute lung injury in canine model of severe salt water drowning. Scand J Trauma Resusc Emerg Med. 2016; 24:40.

34. Emans ME, van der Putten K, van Rooijen KL, Kraaijenhagen RJ, Swinkels D, van Solinge WW, et al. Determinants of red cell distribution width (RDW) in cardiorenal patients: RDW is not related to erythropoietin resistance. J Card Fail. 2011; 17(8):626-33.

35. Zhang L, Yu CH, Guo KP, Huang CZ, Mo LY. Prognostic role of red blood cell distribution width in patients with sepsis: a systematic review and meta-analysis. BMC Immunol. 2020; 21(1):40.

36. Jansma G, de Lange F, Kingma WP, Vellinga NA, Koopmans M, Kuiper MA, Boerma EC. 'Sepsis-related anemia' is absent at hospital presentation; a retrospective cohort analysis. BMC Anesthesiol. 2015; 15:55.

37. Jakacka N, Snarski E, Mekuria S. Prevention of latrogenic Anemia in Critical and Neonatal Care. Adv Clin Exp Med. 2016; 25(1):191-7.

38. Rodriguez RM, Corwin HL, Gettinger A, Corwin MJ, Gubler D, Pearl RG. Nutritional deficiencies and blunted erythropoietin response as causes of the anemia of critical illness. Journal of critical care. 2001; 16(1):36-41.

\section{Tables}

\section{Table 1. Baseline characteristics}




\begin{tabular}{|c|c|c|c|}
\hline & $\begin{array}{l}\text { Control group } \\
(n=50)\end{array}$ & $\begin{array}{l}\text { CVVH group } \\
(n=39)\end{array}$ & $P$ value ${ }^{a}$ \\
\hline Age $\varangle$ years $\rrbracket$ & $76.0(68.2,85.1)$ & $74.5(65.0,83.3)$ & 0.489 \\
\hline $\operatorname{Male}[n(\%)]$ & $29(58.0 \%)$ & $18(46.2 \%)$ & 0.292 \\
\hline \multicolumn{4}{|l|}{ Sites of infection $[n(\%)]$} \\
\hline Lung & $21(42.0 \%)$ & $12(30.8 \%)$ & 0.377 \\
\hline Abdomen & $13(26.0 \%)$ & $17(43.6 \%)$ & 0.114 \\
\hline Biliary system & $9(18.0 \%)$ & $6(15.4 \%)$ & 0.784 \\
\hline Soft skin tissue & $9(18.0 \%)$ & $4(10.2 \%)$ & 0.375 \\
\hline \multicolumn{4}{|l|}{ Comorbidities [ $n(\%)]$} \\
\hline Hypertension & $28(56.0 \%)$ & $20(51.3 \%)$ & 0.675 \\
\hline Diabetes mellitus & $20(40.0 \%)$ & $11(28.2 \%)$ & 0.271 \\
\hline Stroke & $1(2.0 \%)$ & $0(0 \%)$ & 1.000 \\
\hline Acute myocardial infarction & $2(4.0 \%)$ & $0(0 \%)$ & 0.502 \\
\hline Deep venous thrombosis & $4(8.0 \%)$ & $1(2.6 \%)$ & 0.380 \\
\hline Post-operative patients [ $n(\%)]$ & $7(14.0 \%)$ & $3(7.7 \%)$ & 0.503 \\
\hline Patients using vasopressors $[n(\%)]$ & $27(54.0 \%)$ & $20(51.5 \%)$ & 0.833 \\
\hline Mechanical ventilation $[n(\%)]$ & $26(52.0 \%)$ & $17(43.6 \%)$ & 0.523 \\
\hline $\mathrm{PaO}_{2} / \mathrm{FiO}_{2}$ & $216.5(175.8,245.0)$ & $217.0(192.0,244.0)$ & 0.579 \\
\hline Procalcitonin (ng/mL) & $7.32(2.45,10.23)$ & $5.00(1.16,16.90)$ & 0.993 \\
\hline Lactate (mmol/L) & $4.05(2.28,5.53)$ & $3.10(1.80,4.90)$ & 0.101 \\
\hline Creatinine $(\mu \mathrm{mol} / \mathrm{L})$ & $132.0(81.8,181.8)$ & $147.0(109.0,195.0)$ & 0.273 \\
\hline Reticulocytes $\left(\times 10^{9} / \mathrm{L}\right)$ & $66.0(43.3,81.3)$ & $50.0(38.0,65.0)$ & 0.083 \\
\hline Mean corpuscular volume (fL) & $88.5(82.0,93.0)$ & $88.0(85.0,93.0)$ & 0.967 \\
\hline Mean corpuscular hemoglobin (pg) & $32.0(30.0,34.0)$ & $32.0(29.0,34.0)$ & 0.800 \\
\hline $\mathrm{MCHC}(\mathrm{g} / \mathrm{L})$ & $334.5(320.8,347.0)$ & $333.0(314.0,348.0)$ & 0.628 \\
\hline SOFA & $9.0(5.0,13.0)$ & $10.0(3.0,14.0)$ & 0.191 \\
\hline
\end{tabular}

Values are the median (interquartile range). 


\section{Figures}

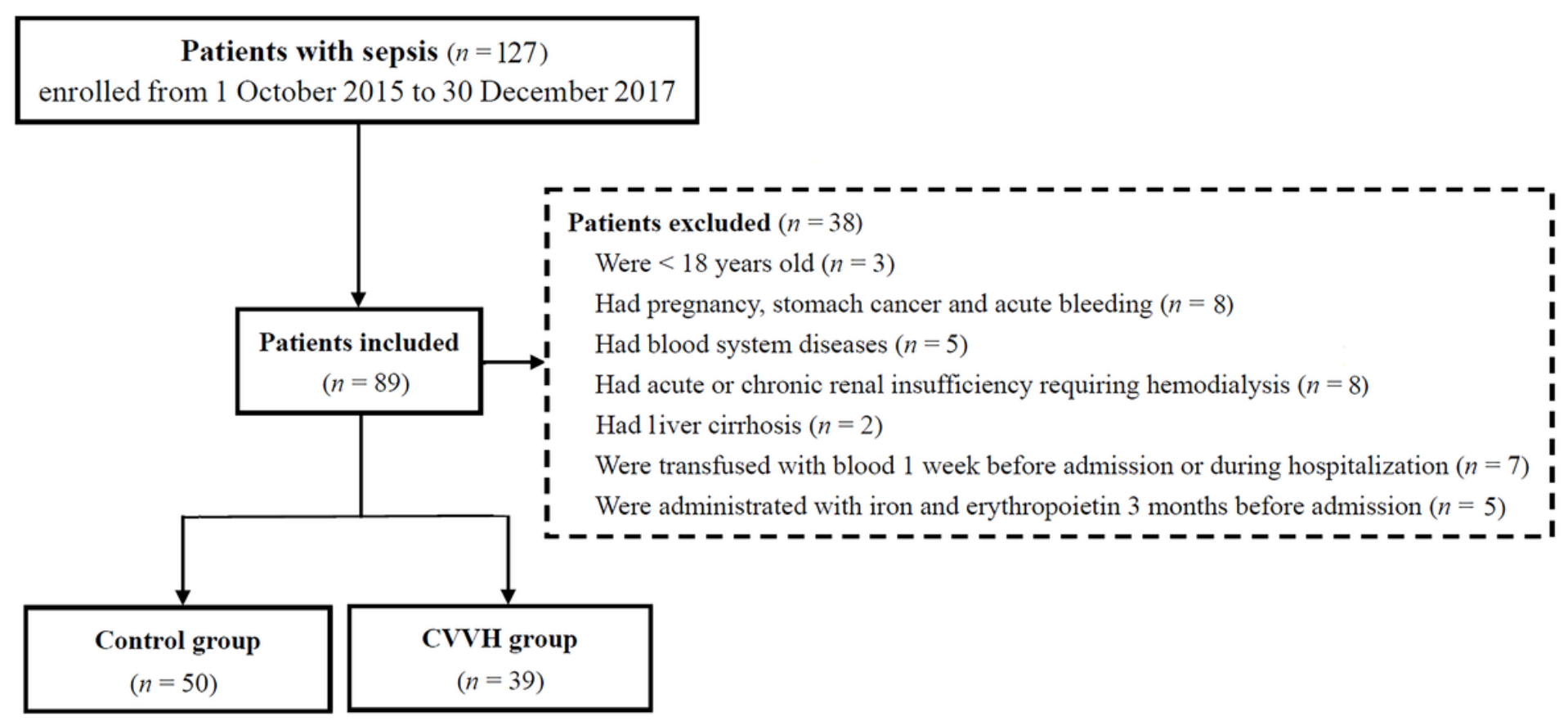

\section{Figure 1}

Study flowchart. CVVH, continuous veno-venous hemofiltration.

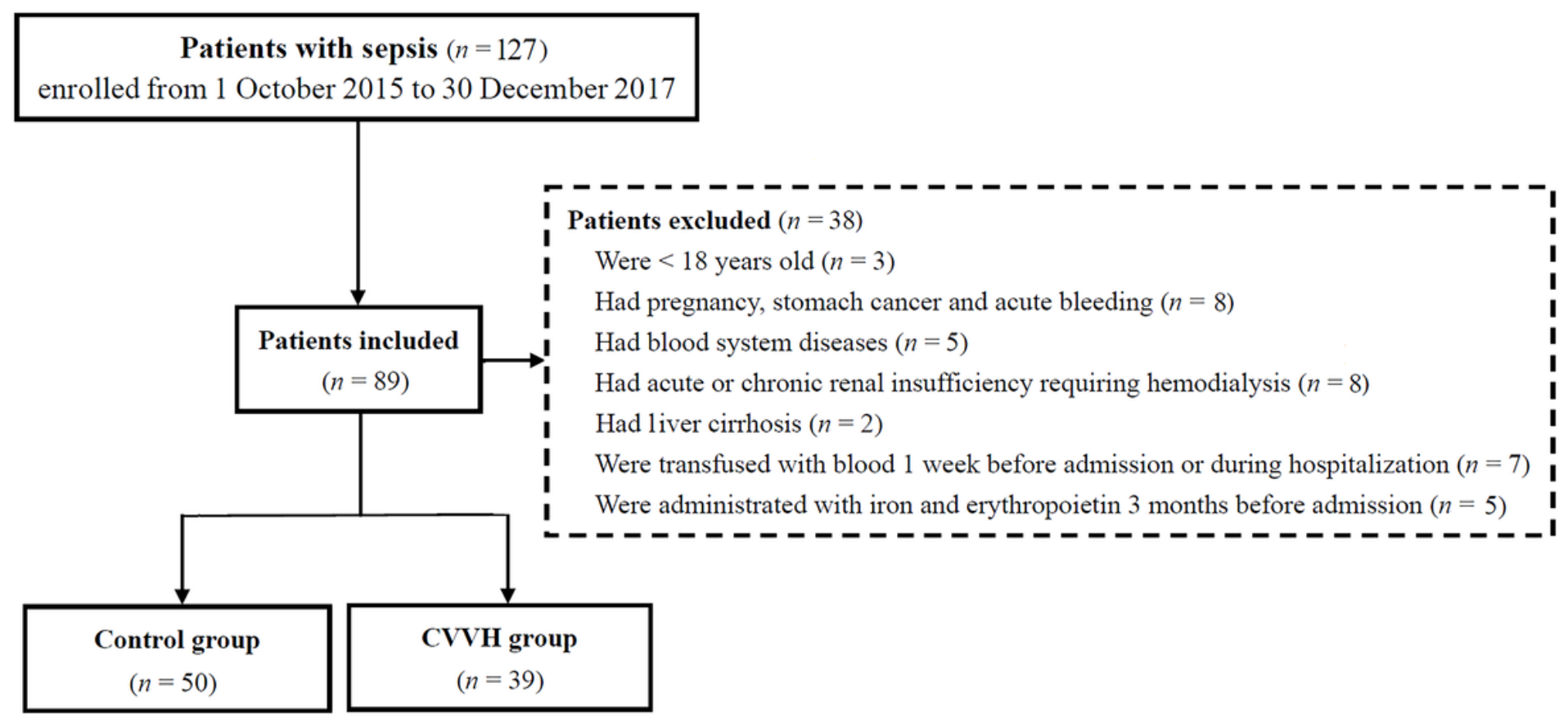


Study flowchart. CVVH, continuous veno-venous hemofiltration.

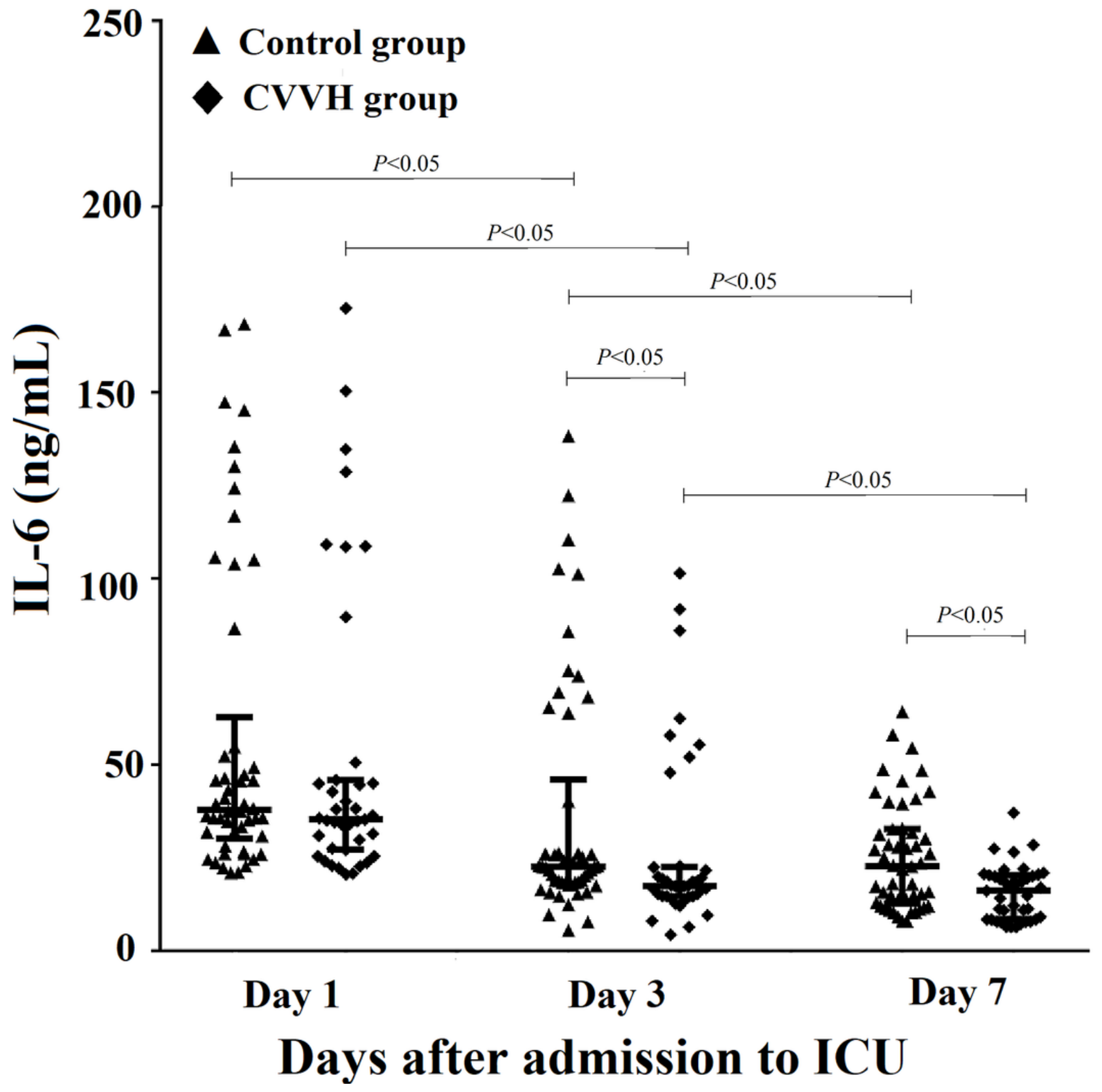

Figure 2

Comparison of plasma IL-6 between the control and CVVH groups. CVVH, continuous veno-venous hemofiltration. 


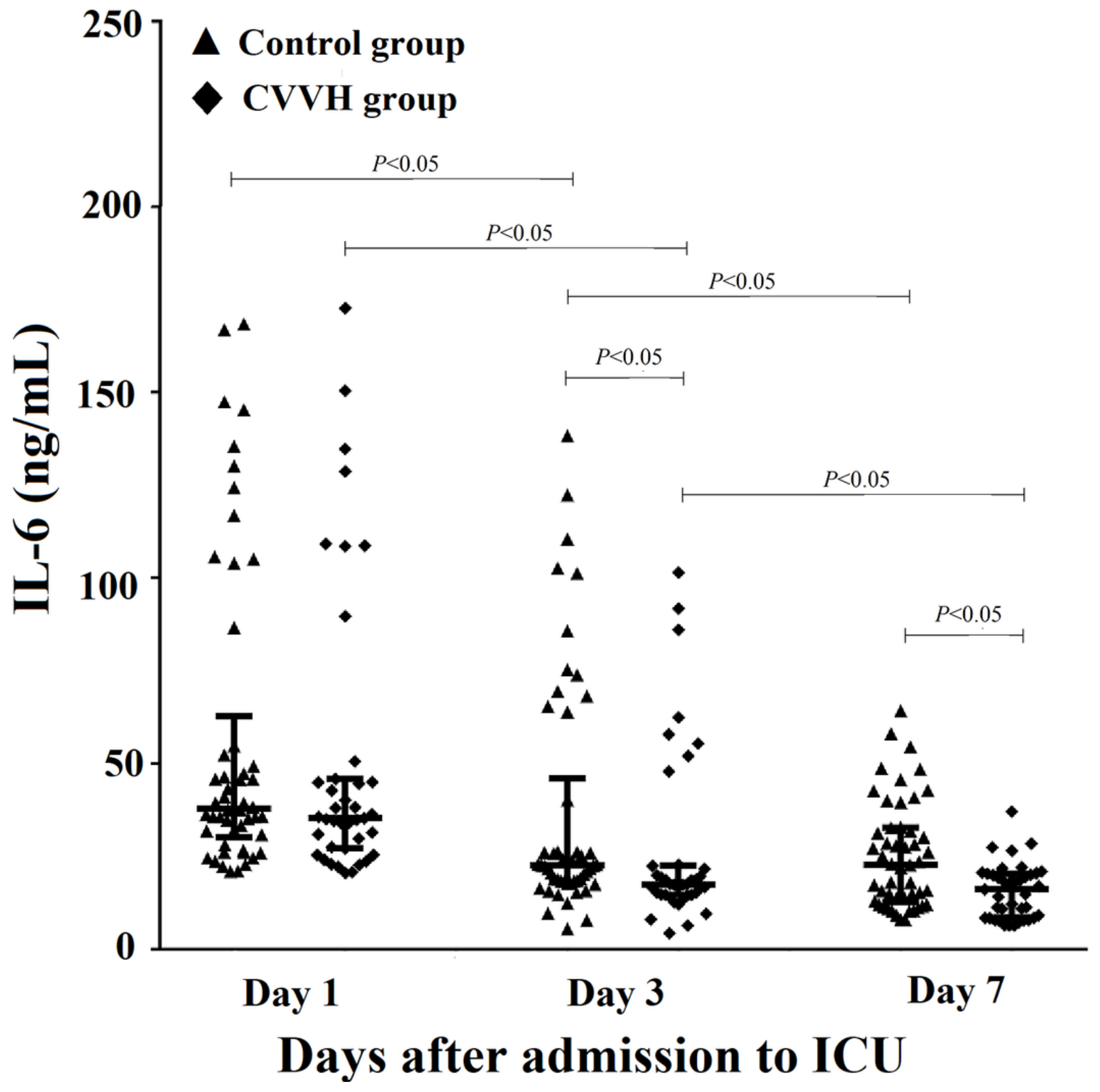

Figure 2

Comparison of plasma IL- 6 between the control and CVVH groups. CVVH, continuous veno-venous hemofiltration. 

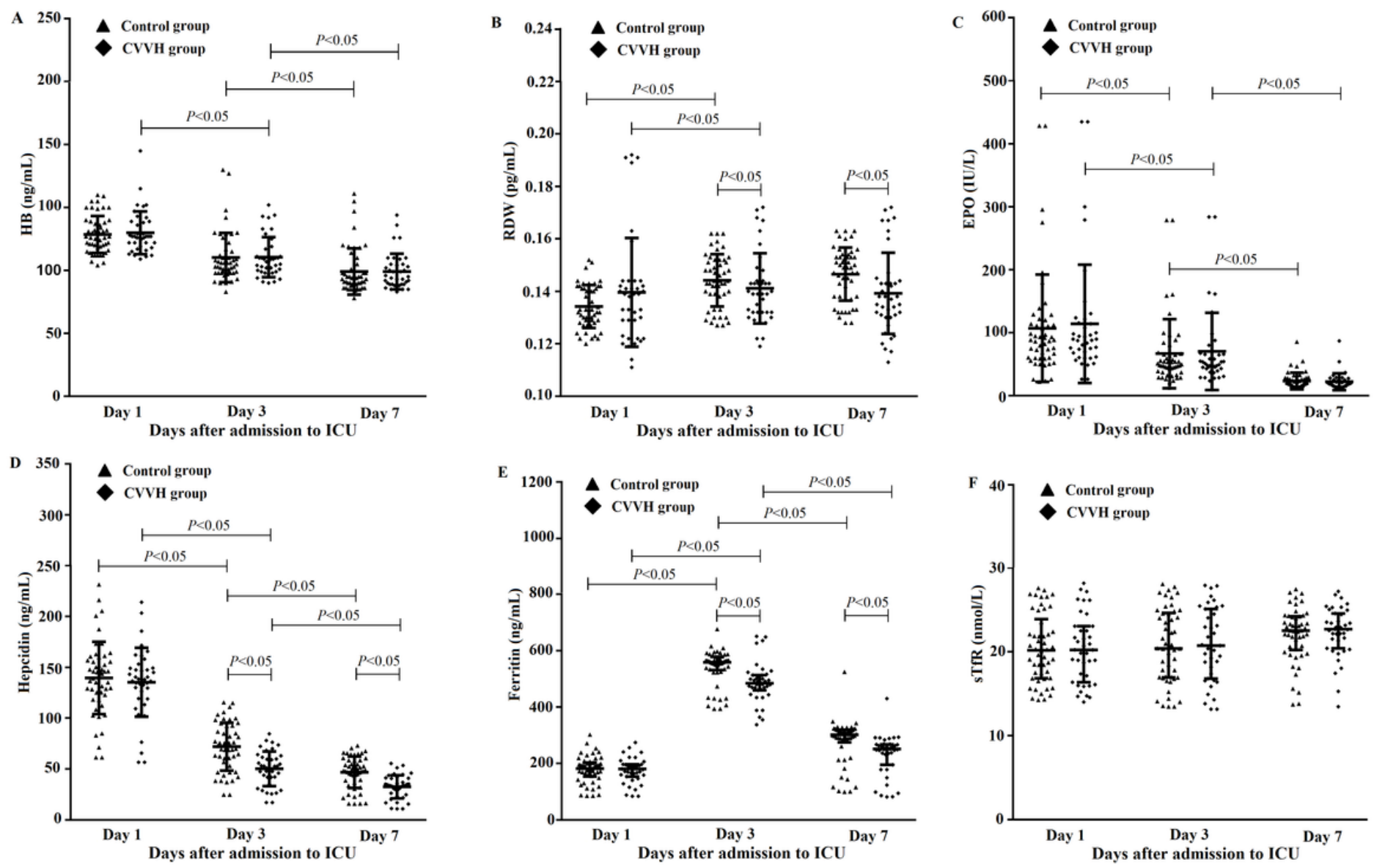

Figure 3

Comparison of $\mathrm{Hb}$, RDW, plasma EPO, hepcidin, ferritin, and sTfR between the control and CVVH groups. $\mathrm{CVVH}$, continuous veno-venous hemofiltration; EPO, erythropoietin; HB, hemoglobin; RDW, red blood cell distribution width; sTfR, soluble transferrin receptor. 

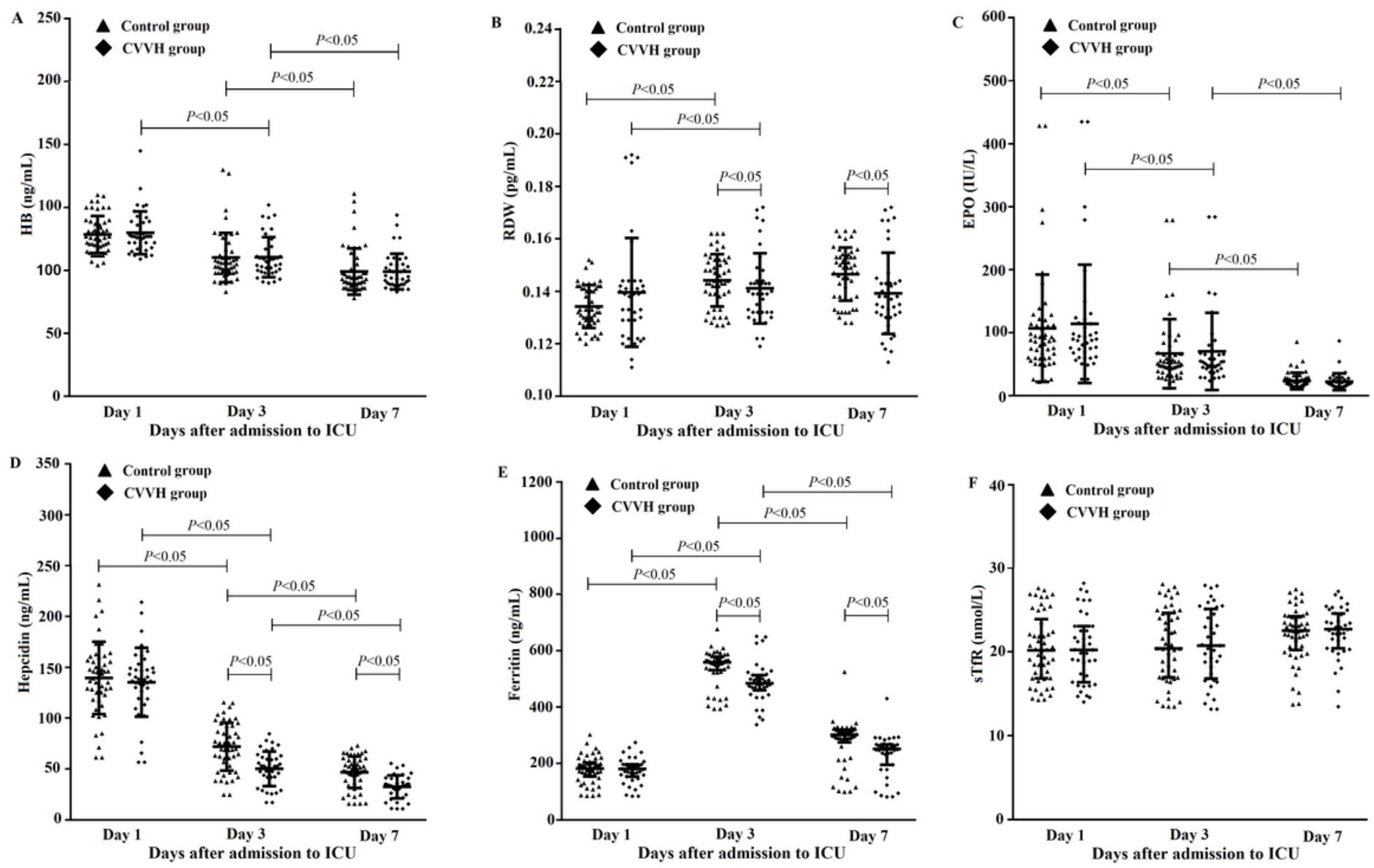

Figure 3

Comparison of $\mathrm{Hb}$, RDW, plasma EPO, hepcidin, ferritin, and sTfR between the control and CVVH groups. $\mathrm{CVVH}$, continuous veno-venous hemofiltration; EPO, erythropoietin; HB, hemoglobin; RDW, red blood cell distribution width; sTfR, soluble transferrin receptor. 


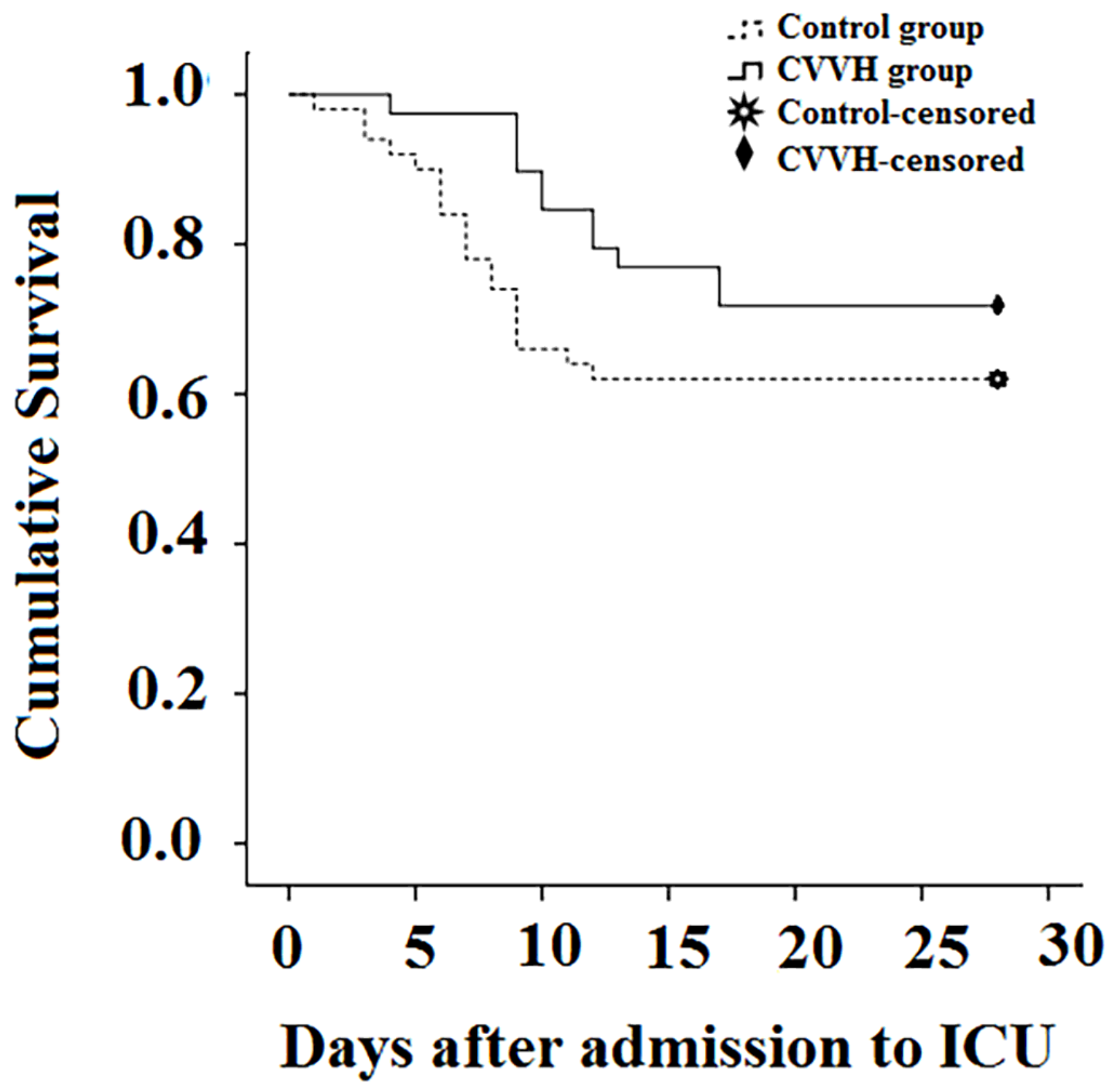

Figure 4

Kaplan-Meier Survival Curves for the control and CVVH groups. The difference in survival curves was not significant between the control and CVVH groups $(P=0.187)$, as compared using a Log rank test. CVVH, continuous veno-venous hemofiltration. 


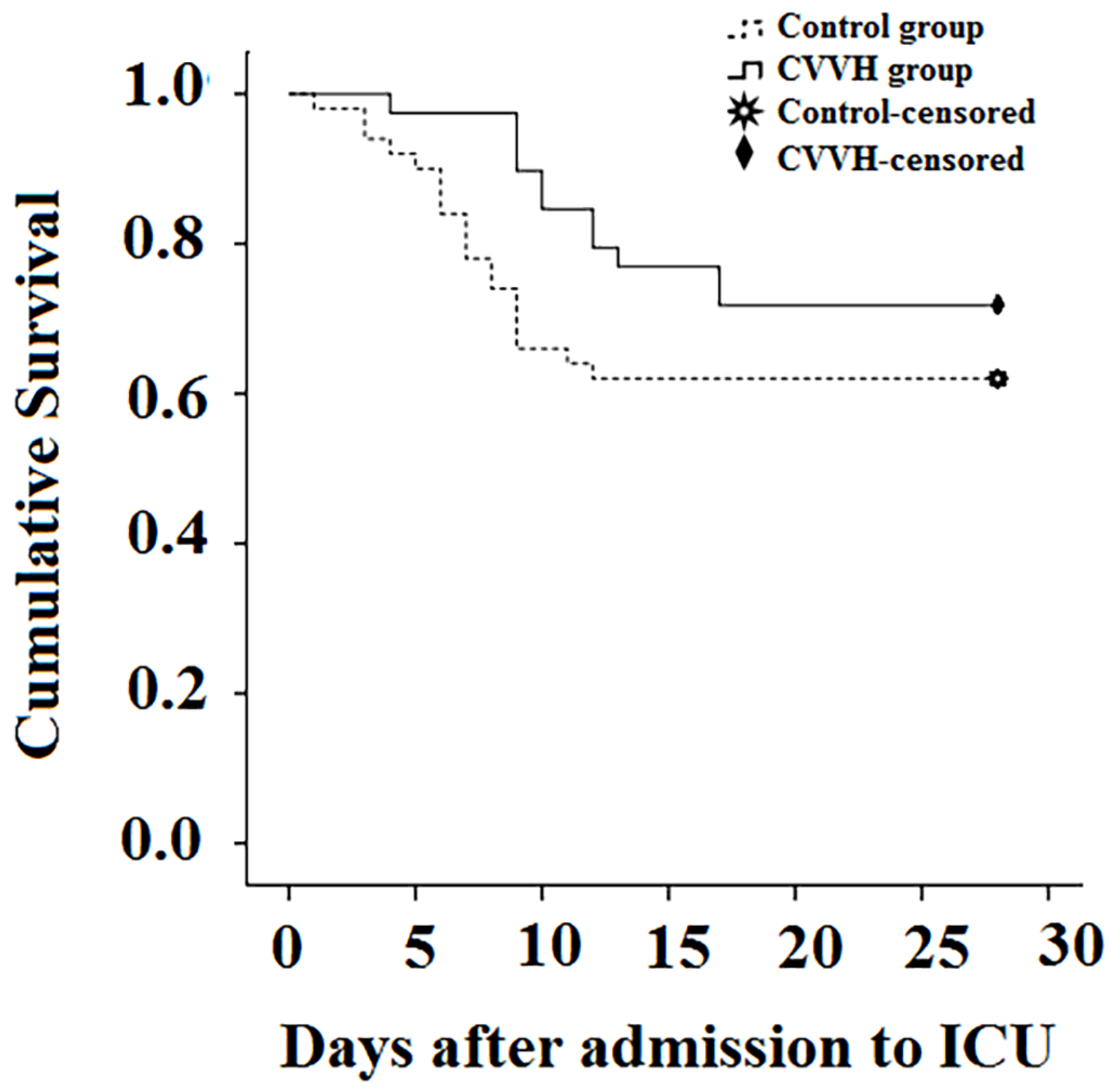

Figure 4

Kaplan-Meier Survival Curves for the control and CVVH groups. The difference in survival curves was not significant between the control and CVVH groups $(P=0.187)$, as compared using a Log rank test. CVVH, continuous veno-venous hemofiltration. 